\title{
Vibration Control Of Steel Frames Using Magnetorheological Dampers: A New Control Algorithm.
}

\author{
G.K. Arunvivek ${ }^{a,}{ }^{,}$, R. Saravanakumar ${ }^{b}$ \\ a Department of Civil Engineering, Sri Shakthi Institute of Engineering \& Technology, Coimbatore, India 641062. \\ b Department of Civil Engineering, K.P.R. Institute of Engineering \&Technology, Coimbatore, India 641407.
}

\section{*Corresponding Author arunvivekgk@siet.ac.in (G.K. Arunvivek)}

Received : 04 04 2019 Accepted: 12 05 2109

\begin{abstract}
Control technologies application to steel structure is mainly anticipated to enhance the structural performance against natural hazards. In particular smart base isolation system connected with semi active isolator at the base with controllable semi devices gaining impulse for its efficiency and economic reasons. Generally the development of control design strategies through system dynamics concept had not been considered entirely for structural applications. Structural characteristics which help to divulge structural properties, hitherto flout by civil engineering circle are assimilated with control techniques to construct indices in modal and nodal coordinates for the endurance of the control action to utilize their fullest capabilities. In this study, an isolated 3D steel frame model is developed. Magneto Rheological dampers are fixed with 3D steel frame model which act as a smart control device. Besides, Force transducers and Piezoresistive Actuator in tandem with Deltatron conditioning amplifier are also used. Presently many techniques are employed for the optimum placement of actuators and sensors in vibration control systems. The concept of controllability observability is used in these methods. The specific relationship between the vibration modes and controllability observability simplifies this approach. This study envisaged the compatibility of force transducers along with triaxial and uniaxial accelerometers fixed at various trial spots on the model structure to quantify the damping force and absolute accelerations of the structure and the dampers individually, positioned in the system, against the excitation of the structure.
\end{abstract}

Keywords: Sensors, Accelerometers, Magnetorheological dampers, Vibration control, Semi - Active Isolation.

\section{Introduction}

In the current scenario, numerous techniques have been developed for the optimal installation of sensors and actuators in vibration control systems. Currently, concept of controllability and observability is ruling the roost in various facets of vibration control. Controllability and observability properties can be shaped by varying the configurations of sensors and actuators. This optimization problem is closely related to achieving high performance with minimal cost especially for steel frames [1 4]. If a system is considered in which sensors and actuators are located near to the nodes of vibration modes may require an exceptional control force, or even may be uncontrollable. This approach is expedited by explicit relationships between controllability and observability and vibration modes, in their approach Hamdan and Nayfeh's introduced a generalized angle between the two vector spaces in that controllability and observability are taken as the left eigenvectors and the input influence matrix as the column vectors, also the right eigenvectors and the output measurement matrix as the column vectors furthermore improved the method by prolonging the results to be used with a balanced coordinate system and adding the magnitude of the measures, the norms of eigenvectors, when used in that coordinate system. A balanced coordinate system is beneficial because it warrants that the system is correspondingly controllable and observable [5 8].

This paper briefly outlines the controllability observability based approach in practical applications. This approach comprises the computation of the system norms of each and every device location for chosen modes, according to their performance in the system norm then they are graded. It agrees with the control objective of the LQR algorithm to be used in this study, whose cost function is actually a 2 norm, and it is comparatively simple if it compared with other algorithms [9 10]. 
In this study, Hankel singular norm method is proposed. The Hankel norm is worthwhile because it reflects both controllability and observability, and is invariant under linear similarity transformations. The placement indices took into consideration for the closed loop effects when the sensors are not fixed at the performance evaluation positions and actuators are not mounted at the critical disturbance location in index normalization. To execute the approach much applicable to civil engineering problems, this investigation contemplates only the case when the sensors are collocated with performances and the actuators are placed with disturbances. The normalization procedure is simplified by this assumption [11 13].

\section{Influences of the Cross Couplings on Norms in the Feedback Loop}

A structure's inputs consist of disturbances as well as control inputs, besides plant outputs comprising controlled outputs and measurements. In engineering applications, control devices and sensors are collocated at accessible location, not necessarily located with the disturbance and outputs used for performance evaluations. It is revealed that cross couplings between the inputs and outputs all effects on the structural norms due to the feedback loop, so it is essential to scrutinize these effects for placement rules by the structural norms. In the first step, a general model of a feedback control system that explicitly includes the desired inputs and outputs is defined.

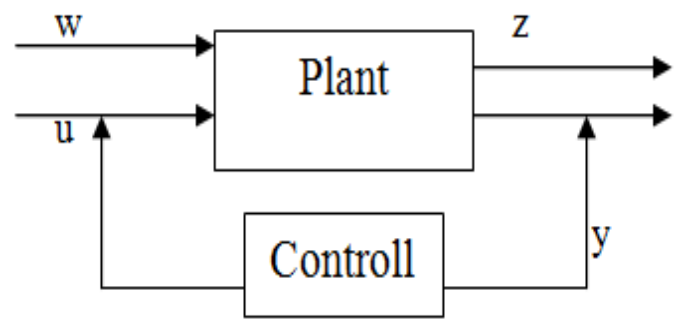

Fig 1. Feedback Control System

In the feedback control system controller produces the control input $u$ to the plant. The output comprises of the measurement output $\mathbf{y}$ and regulated output $\mathbf{z}$. The feedback loop is closed between the controller (actuator) and measurement output. By and large, the measurement output is diverse from the regulated output, though they are identical in certain applications. The state model of the plant for the closed loop system is illustrated in Fig.1

$$
\begin{gathered}
\dot{x}(t)=\mathrm{Ax}(t)+[\mathrm{B} E]\left[\begin{array}{c}
\mathrm{u}(\mathrm{t}) \\
\mathrm{w}(\mathrm{t})
\end{array}\right] \\
{\left[\begin{array}{l}
z(t) \\
y(t)
\end{array}\right]_{=}\left[\begin{array}{l}
C_{z} \\
C_{y}
\end{array}\right]_{\mathrm{x}(t)+}\left[\begin{array}{ll}
D_{z} & E_{Z} \\
D_{y} & E_{y}
\end{array}\right]\left[\begin{array}{l}
u(t) \\
w(t)
\end{array}\right]}
\end{gathered}
$$

$\mathbf{G}_{\mathrm{wz}}$ is the transfer function matrix from $\mathbf{w}$ to $\mathbf{z}, \mathbf{G}_{\mathrm{wy}}$ be the transfer function $\mathbf{w}$ to $\mathrm{y}, \mathbf{G}_{\mathrm{uz}}$ be the transfer function matrix from $\mathbf{u}$ to $\mathbf{z}$, and $G_{\mathbf{u y}}$ be transfer function matrix from $\mathbf{u}$ to $\mathbf{y}$. These open loop transfer functions are expressed by

$$
\begin{aligned}
& \mathrm{G}_{\mathrm{uz}}(\mathrm{s})=\mathrm{C}_{\mathrm{Z}}(\mathrm{sI} \sim \mathrm{A})^{-1} \mathrm{~B}+\mathrm{D}_{\mathrm{Z}} \\
& \mathrm{G}_{\mathrm{uy}}(\mathrm{s})=\mathrm{C}_{\mathrm{y}}(\mathrm{sI} \sim \mathrm{A})^{-1} \mathrm{~B}+\mathrm{D}_{\mathrm{y}} \\
& \mathrm{G}_{\mathrm{wz}}(\mathrm{s})=\mathrm{C}_{\mathrm{Z}}(\mathrm{sI} \sim \mathrm{A})^{-1} \mathrm{E}+\mathrm{E}_{\mathrm{z}} \\
& \mathrm{G}_{\mathrm{wy}}(\mathrm{s})=\mathrm{C}_{\mathrm{y}}(\mathrm{sI} \sim \mathrm{A})^{-1} \mathrm{E}+\mathrm{E}_{\mathrm{y}}
\end{aligned}
$$

The closed loop transfer function from $\mathbf{w}$ to $\mathbf{z}$ then becomes

$$
G_{w z-c l}=G_{u z}\left(I \sim G_{c y} G_{u y}\right)^{-1} K G_{w y}+G_{w z} \text {. }
$$

Equation (4) indicates that the controller affects the closed loop performance through the action from $\mathbf{u}$ to $\mathbf{y}$ along with the cross actions from $\mathbf{u}$ to $\mathbf{z}$ and $\mathbf{w}$ to $\mathbf{y}$. However the transfer function matrics $\mathbf{G}_{\mathrm{wy}}$ or $\mathbf{G}_{\mathrm{uz}}$ were zero, the controller could not influence the response. Consequently, for non collocated systems, the sensor and actuator connectivity $\mathbf{G}_{\mathrm{uy}}$ is not the only factor that determines the closed loop performance. This makes the placement problem complicated because the above effort would be in vain if $\mathbf{G}_{\mathrm{wy}}$ or $\mathbf{G}_{\mathrm{uz}}$ decreases while the prominence of location (placement indices) is identified by large $\mathbf{G}_{\text {uy. }}$ Denote subscript $i$ for the $i^{\text {th }}$ mode, the following multiplicative property of modal norms holds

$$
|| \mathbf{G}_{\mathrm{wz}, \mathrm{i}}|||| \mathbf{G}_{\mathrm{uy}, \mathrm{I}}|| \cong|| \mathbf{G}_{\mathrm{wy}, \mathrm{I}}|||| \mathbf{G}_{\mathrm{uz}, \mathrm{I}}|| \quad \text { Eq.(5) }
$$

Where ||$.||$ denotes either $H_{2}, H^{\infty}$, or Hankel norms, and subscript I denotes the $i^{\text {th }}$ mode.

This property can be determined directly using the imprecise relationship among the transfer functions. This property betokens that for each mode the product of norms of the performance loop (from disturbance to response) and the control loop (from actuators to sensor response) is nearly identical to the output of the norms of cross couplings concerning the disturbance and sensors, and between the actuators and performance. It also indicates that improvement in $\mathbf{G}_{u y}$ automatically leads to improvement in

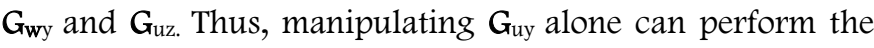
actuator and sensor location problems. This outcome is essential for the placement problem.

Equation. (4) Signify the Laplace transforms of the transforms of the $\mathrm{y}, \mathrm{z}, \mathrm{u}$ and $\mathrm{w}$ vectors with capital letters. Then the transfer function of the plant is

$$
\begin{aligned}
& {\left[\begin{array}{l}
\mathrm{Z} \\
\mathrm{Y}
\end{array}\right]_{=\mathrm{G}(\mathrm{s})}\left[\begin{array}{c}
\mathrm{U} \\
\mathrm{W}
\end{array}\right]_{=}\left[\begin{array}{cc}
\mathrm{G}_{\mathrm{uz}} & \mathrm{G}_{\mathrm{wz}} \\
\mathrm{G}_{\mathrm{uy}} & \mathrm{G}_{\mathrm{wy}}
\end{array}\right]\left[\begin{array}{c}
\mathrm{U} \\
\mathrm{W}
\end{array}\right]=} \\
& {\left[\begin{array}{ll}
\mathrm{G}_{\mathrm{uz}} \mathrm{U}+\mathrm{G}_{\mathrm{wz}} & \mathrm{W} \\
\mathrm{G}_{\mathrm{uy}} \mathrm{U}+\mathrm{G}_{\mathrm{wy}} & \mathrm{W}
\end{array}\right]}
\end{aligned}
$$


The transfer function of the controller is

$\mathrm{U}=\left[\begin{array}{ll}\mathrm{G}_{\mathrm{cr}} & \mathrm{G}_{\mathrm{cy}}\end{array}\right]\left[\begin{array}{l}\mathrm{R} \\ \mathrm{Y}\end{array}\right]_{=\mathrm{G}_{\mathrm{cr}} \mathrm{R}+\mathrm{G}_{\mathrm{cy}} \mathrm{Y} .}$

Replacing Y from the equation (4) into above equation yields $\mathrm{U}=\left(\mathrm{I}-\mathrm{G}_{\mathrm{cy}} \mathrm{G}_{\mathrm{uy}}\right)^{-1} \mathrm{G}_{\mathrm{cy}} \mathrm{G}_{\mathrm{wy}} \mathrm{W}$.

Substituting equation (6) into the first equation yields the closed-loop transfer function from $\mathrm{w}$ to $\mathrm{z}$ of the feedback control system.

$Z=\left(G_{u z}\left(I-G_{c y} G_{u y}\right)^{-1} G_{c y} G_{w y}+G_{w z}\right) W$.

If $G_{c y}=K(s)$, the transfer function diagram is shown in Fig 2.

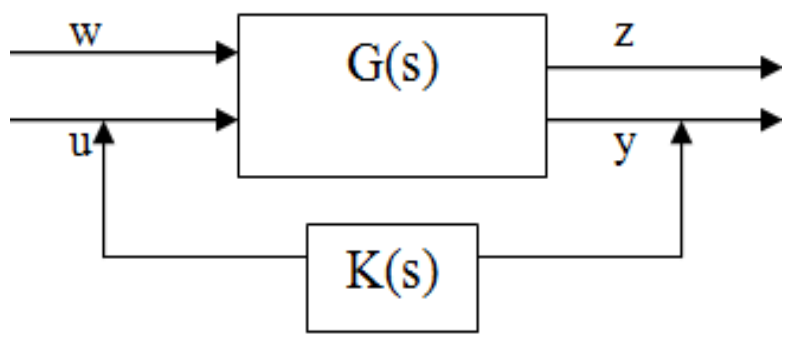

Fig .2. Diagram of a Constant - Gain Feedback Control System

\section{Placement Indices}

To delineate the controllability absorbability based sensor and actuator location model, information about the location and size of the actuator is in the control input influence matrix $\mathrm{B}$. Information about the sensor location is contained in the matrix $C$ is needed. The placement strategy here only considers the case that actuators are collocated with the disturbance, and sensors are collocated with the performance outputs.

For this benchmark problem, control devices are required to be placed at base level and conveniently, at bearing locations. So there are candidate locations for control devices. Accelerometers may be fixed at the four corners of each floor including the base. Each corner has one accelerometer in the $\mathrm{x} \sim$ and one in the $\mathrm{y} \sim$ direction, giving twelve available accelerometer locations for each floor. Note that three sensors would be enough for each floor to capture the responses because each floor has three DOFs. Thus, the problem of placement is to determine a reasonable subset of locations for control devices that offer high controllability of the desired modes, and a reasonable subset of sensors that offer high absorbability in the detection of the desired modes.

For each mode, the Hankel norm with a set of actuators or sensors is the rms sum of the Hankel norm with each single actuator or sensor from this set, i.e.,

$$
r_{i}=\sqrt{\sum_{j=1}^{s} \gamma_{i j}^{2}} \text { or } \gamma_{i}=\sqrt{\sum_{k=1}^{r} \gamma_{i k}^{2}}
$$

Finally, the Hankel norm of the system is the largest norm of its mode, i.e.,

$$
\left.\left.|| G\right|_{\mathrm{h}} \cong \max _{i}|| G\right|_{\mathrm{h}_{\mathrm{h}}}=\gamma_{\max }=\left.0.5|| G\right|_{\infty} . \quad \text { Eq. (10) }
$$

Where, $\gamma_{\max }$ is the largest Hankel singular value of the system. Equations 9 and 11 provide a means to normalize the indices using Hankel norms so that the indices are between 0 and 1 . For actuator placement, the index $\sigma_{i j}$ that evaluates the $j$ th actuator at the $i$ th mode concerning Hankel norm is defined for all modes and control devices as

$$
\sigma_{i j}=\frac{\left\|G_{i j}\right\|_{h}}{\|G\|_{h}} \text {. }
$$

Similarly, in the sensor placement, the placement index that evaluates the $k$ th sensor at the ith mode is defined as

$$
\sigma_{\mathrm{ij}}=\frac{\left\|G_{i k}\right\|_{h}}{\|G\|_{h}} .
$$

Locations in the neighborhood are not necessarily the best choice because the performance gains achieved using devices at these locations can also be achieved by appropriate gain adjustments (Gawronski, 1998). The best strategy is to find locations that cannot be compensated for by gain adjustment. Naturally, correlation coefficients are used to remove highly correlated locations.

Define a vector of the squares of the ith Hankel modal norms,

$$
g_{\mathrm{i}}=\left[\begin{array}{c}
\left\|G_{i j}\right\|_{h}^{2} \\
\left\|G_{i 2}\right\|_{h}^{2} \\
\vdots \\
\left\|G_{i n}\right\|_{h}^{2}
\end{array}\right]
$$

where, $\left.|| G_{i k}\right|_{h}$ is the Hankel norm of the $k^{\text {th }}$ mode at the $i^{\text {th }}$ control device or sensor. The correlation coefficient $\rho_{i k}$ is defined as

$\rho_{i k}=\frac{g_{i}^{T} g_{k}}{\left\|g_{i}\right\|_{2}\left\|g_{k}\right\|_{2}}, i=1, \ldots, r, k=i+1, \ldots, r$. Eq. (15)

Given a small positive number $\varepsilon$, say $\varepsilon=0.001$, denote the membership index $\mathrm{I}(\mathrm{k}), k=1, \ldots, r$, where $r$ is the number of sensors (control devices). This index is determined as 


$$
\mathrm{I}(\mathrm{k})=\left\{\begin{array}{lc}
0 & \rho_{\mathrm{ik}} \\
1 & \text { elsewhere }
\end{array}\right.
$$$$
\text { and } \rho_{k}<\rho_{i} \text { for } k>i \text {. }
$$

If $\mathrm{I}(\mathrm{k})=1$, then the $k$ th sensor (actuator) is accepted. If I $(k)=0$, the $k$ th sensor (actuator) is rejected. In the case of I $(\mathrm{k})=0$, the two locations $i$ and $k$ are either highly correlated $\left(\rho_{i k}>1 \sim \varepsilon\right)$, or the ith location has a higher performance $\sigma_{i}$.

Based on the above analysis the placement strategy is established. For this 3D base isolation benchmark problem, sensor placement is more flexible, so actuator locations are decided first. The procedure is described as follows:

- Place the control devices in order at the bearing locations, one in the $x \sim$ direction and one in the $y^{\text {r }}$ direction. Assume each admissible sensor location has two sensors, one in the $x \sim$ and one in the $y \sim$ direction, so that the $C_{m}$ matrix is fixed. For each location, compute the modal $\mathrm{B}_{\mathrm{m}}$ matrix and then the Hankel placement indices for all modes, until the $42 \times 12$ (total 27 modes) placement index matrix is formed.

- Roughly choose 20 25 locations with the largest placement indices in the lower modes.

- Check the correlation coefficients for the selected locations. Reject actuators with $\mathrm{I}(\mathrm{k})=0$. The resulting values (say, 10) are the final locations. Wherever the number is lesser than 10, more locations should be added in step2; if the number is greater than 10 , reduce the locations, so that rejection condition is stricter.

- Fix the $B_{m}$ matrix for resulting set of actuator locations. Compute the floor sensor placement indices, assuming sensors are put at all four corners on this floor while none are on other floors to determine $\mathrm{C}_{\mathrm{m}}$ matrix. Repeat for each floor until the $9 \times 27$ placement index matrix is formed.
- Reject insignificant floors that have very low sensor placement indices.

- For the remaining floors, compute the corner plane indices one by one. Retain the non correlated corners.

All control device and sensor locations are thus determined, following the above procedure.

4. Control device and sensor placement for the benchmark problem

The 3D dynamics of the benchmark problem have, the parameters of the superstructure are known. The optimal isolation parameters, bearing stiffness and damping coefficient of the rheological dampers, have been determined. The experimental setup used in this study is shown in the Fig.3. There are four corners, and thus eight available locations for accelerometers for each floor, some of which are redundant. Three accelerometers per floor (6 total accelerometers) would provide a measure of all motions of that particular floor. So the following step is to compute the corner indices of floors 3 to 8 . Place two accelerometers (One in the $\mathrm{x}$-direction and one in the $\mathrm{y}$-direction) at each corner of floor 3 and compute the indices and then repeat this procedure for the remaining floors. In order to evaluate the performance with the reduced set of sensors, comparisons were performed for responses of the isolated benchmark building.

The control algorithm is chosen as LQG, and MR dampers are adopted as the control devices to examine the performance of these systems. Weights are placed on the corner base drifts, corner base accelerations, and corner top floor acceleratighs $\left(q_{\text {drift }}=4.642 \times S_{v_{i}} 0^{8}, q_{\text {accelration }}=1.145 \times\right.$ $10^{9}, \mathrm{R}=\mathrm{I}_{20 \times 20}, \quad x_{g} x_{g}=25 \mathrm{I}_{2}$, and $v_{i} v_{i}=\mathrm{I}_{\mathrm{ns} \times 20}$, where $n s$ is the number of sensors). Noise in the sensors is simulated by adding a band limited white noise to each signal that is scaled to have an RMS of approximately $3 \%$ of the corresponding maximum RMS responses of the passive system.

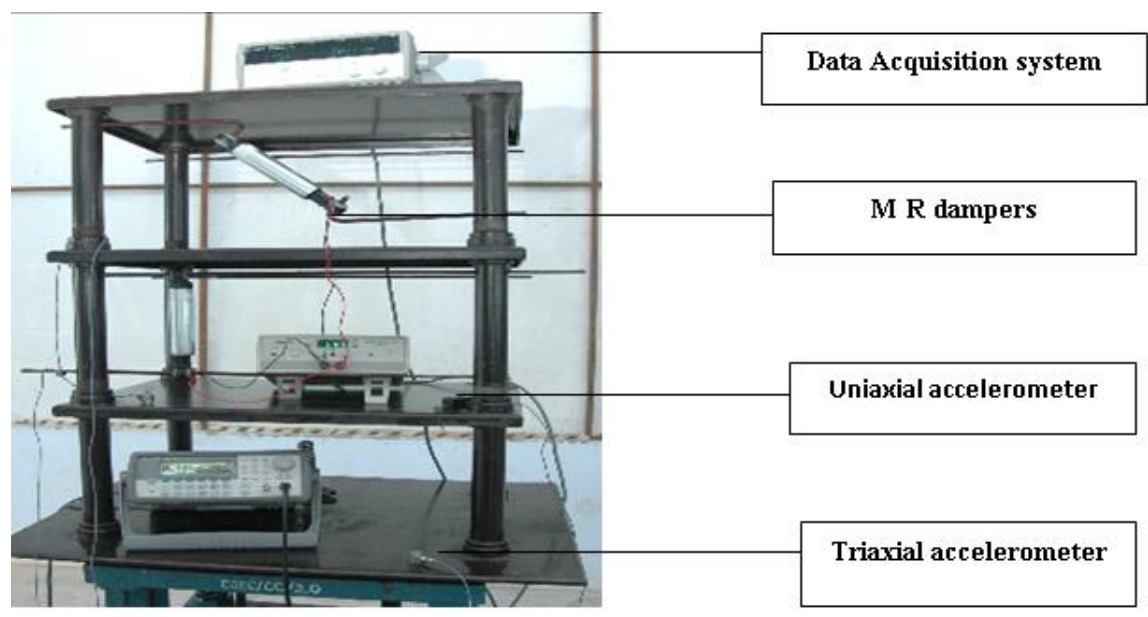

Fig .3 Accelerometer and transducer placement in steel frame 
Time history responses of the base drift, inter story drift between the II and III floors, and roof accelerations at corner 1 in the $x$-direction for full sensor placement and reduced sensor placement are measured. It was observed that the response values are in close proximity and differences in the resulting performance of the two systems are not substantial.

\section{Conclusion}

A controllability/observability -based approach has been proposed to place control devices and sensors effectively. The placement indices are based on Hankel singular values, which are invariant for both unbalanced and balanced systems. Validation of the technique for control device (MR dampers) not collocated with disturbances, correlations between locations are examined to avoid duplication of control effort, and locations with high indices and high correlations are rejected. Seismic responses confirm the efficacy of the reduced set of sensors, Simulated with structural excitation using Dynamic signal analyzer.

\section{References}

[1] H.L. Hsu and H. Halim, Improving seismic performance of framed structures with steel curved Dampers, Eng. Stru., 130 (2017) 99-111.

[2] Jinkoo Kim and Hyungjun Shin, Seismic loss assessment of a structure retrofitted with slit friction hybrid Dampers, Eng. Stru. 130 (2017) 336-350.

[3] R.M. Hessabi and O. Mercan, Investigations of the application of gyro mass dampers with various types of supplemental dampers for vibration control of building structures, Eng. Stru. 126 (2016) 174-186.

[4] J. Kim and S. Kim, Performance-based seismic design of staggered truss frames with friction dampers, Thin Walled Stru., 111 (2017) 197-209.

[5] K. Moon Kwak, and Dong Ho Yang, Dynamic modelling and active vibration control of a submerged rectangular plate equipped with piezoelectric sensors and actuators, J. Fluid. Struct., 54 (2015) 848-867.

[6] G. Cazzulani, S. Cinquemani, L. Comolli, A. Gardella and F. Resta, Vibration control of smart structures using an array of fiber Bragg grating sensors, Mechatronics, 24 (2014) 345-353.

[7] I.F. Lazar, S.A. Neild and D.J. Wagg, Using an inerter based device for structural vibration suppression, Earthquake Eng. Struct. Dynam., 43 (2014) 1129 1147.

[8] K.A.A. Saif, K.A. Aldakkan and M.A. Foda, Modified liquid column damper for vibration control of structures, Int. J. Mech. Sci., 53 (2011) 505-512.
[9] K. Minagawa, T. Watanabe, M. Munakata and K. Koyama, A Novel Apparatus for Rheological Electro Measurements of Magneto Rheological Fluids, J. Non. newton. Fluid. Mechanics. 52 (1994) 59 67.

[10]A.R. Mehrabian and A. Yousefi Koma, A novel technique for optimal placement of piezoelectric actuators on smart structures, J. Franklin Inst. 348 (2011) 12-23.

[11] Y. Fu, H. Di and R. Liu, Light intensity modulation fiber optic sensor for curvature measurement, Opt. Laser Technol., 42 (2010) 594-599.

[12]X.H. Zhou, Y.J. He, L. Xu, and Q.S. Zhou, Experimental study and numerical analyses on seismic behaviors of staggered truss system under low cyclic loading, Thin Walled Struct., 47 (2009) 1343-1353.

[13]S. Hurlebaus and L. Gaul, Smart structure dynamics, Mech. Syst. Signal Process., 20 (2006) 255-281.

\section{About The License}

(C) 2019 The Authors. This work is licensed under a Creative Commons Attribution 4.0 International License which permits unrestricted use, provided the original author and source are credited. 